The authors recommend that physicians consider prescribing ACE inhibitors and ARBsat a low dose and with careful monitoring of kidney function and serum electrolytes to minimize the risk of acute renal failure and hyperkalemia-in all patients with CHF, irrespective of their renal function. Further investigation is needed in the subset of patients on dialysis.

Original article Berger AK et al. (2007) Angiotensinconverting enzyme inhibitors and angiotensin receptor blockers in patients with congestive heart failure and chronic kidney disease. Am Heart J 153: 1064-1073

\section{Trimetazidine reduces risk of contrast-induced nephropathy after coronary procedures}

Contrast-induced nephropathy (CIN), a serious complication of coronary angiography, is particularly common in patients with renal insufficiency. Hydration with isotonic saline is the only generally accepted way of preventing $\mathrm{CIN}$ at present. As formation of oxygen free radicals and renal medullar ischemia are thought to be involved in the pathogenesis of CIN, Onbasili et al. investigated whether administration of trimetazidine, an agent with anti-ischemic and antioxidant properties, might be a useful adjunct to isotonic saline.

The study enrolled 82 patients with mild renal insufficiency who underwent coronary procedures. Patients were randomly assigned to a trimetazidine group ( $20 \mathrm{mg}$ thrice daily orally for $72 \mathrm{~h}$ starting $48 \mathrm{~h}$ before the procedure; $n=40)$ or a control group $(n=42)$. All patients received standard parenteral hydration (isotonic saline $1 \mathrm{ml} / \mathrm{kg} / \mathrm{h}$ from $12 \mathrm{~h}$ before the procedure to up to $12 \mathrm{~h}$ after). The same contrast agent-iopromide-was used in all patients.

$\mathrm{CIN}$ (defined as an absolute serum creatinine increase of $44 \mu \mathrm{mol} / /[0.5 \mathrm{mg} / \mathrm{dl}]$ or a relative increase of $25 \%$ from baseline 1-2 days after the procedure) was significantly more common in controls than in trimetazidine-treated patients (16.6\% vs $2.5 \%$; $P<0.05)$. In controls, serum creatinine levels were higher and creatinine clearances were lower 2 days after the procedure than at baseline; these parameters returned to baseline levels by day 7. By contrast, no changes from baseline in serum creatinine levels or creatinine clearances were seen on day 2 in trimetazidine-treated patients, and by day 7 these patients actually had decreased serum creatinine levels and increased creatinine clearances. No trimetazadine-related adverse effects were noted.

Original article Onbasili AO et al. (2007) Trimetazidine in the prevention of contrast-induced nephropathy after coronary procedures. Heart 93: 698-702

\section{Alemtuzumab induction in kidney transplant recipients}

Two recent Transplantation papers have investigated use of alemtuzumab (Campath ${ }^{\circledR}$; Genzyme Corporation, Cambridge, MA) as an induction agent in recipients of renal allografts.

Pascual et al. retrospectively investigated whether alemtuzumab induction increased rates of glomerular disease recurrence. They included 443 kidney transplant recipients who had experienced glomerulopathies before transplantation; 161 patients received induction therapy with alemtuzumab, 217 with an interleukin 2 receptor antagonist, and 65 with an antithymocyte antibody. Risk of glomerular disease recurrence was similar in patients receiving alemtuzumab and those receiving interleukin 2 receptor antagonists, and was slightly lower in patients receiving an antithymocyte antibody. Graft and patient survival were similar in all groups.

In an open-label trial, Thomas et al. prospectively investigated use of a tolerogenic induction protocol of alemtuzumab in kidney transplant patients at high risk of rejection (defined as those with panel reactive antibody $>20 \%$ or previous failed renal transplant). Patients were randomized to either standard antithymocyte antibody induction followed by triple-drug maintenance therapy (controls; $n=8$ ) or tolerogenic induction with single-dose alemtuzumab followed by maintenance tacrolimus monotherapy $(n=11)$. At 1 year, just one (control) patient had died, and graft survival was similar in the control and alemtuzumab groups ( $87.5 \%$ vs $85.7 \%$ ). Mean serum creatinine levels in patients with surviving allografts were similar in the groups over the course of 12 months, as were rejection and infection rates.

\footnotetext{
Original articles Pascual J et al. (2007) Alemtuzumab induction and recurrence of glomerular disease after kidney transplantation. Transplantation 83: 1429-1434

Thomas PG et al. (2007) Alemtuzumab (Campath 1H) induction with tacrolimus monotherapy is safe for high immunological risk renal transplantation. Transplantation 83: 1509-1512
} 